\title{
Sustainability in bridge construction processes
}

\author{
Pedro Pacheco $\cdot$ António P. Adão da Fonseca • \\ André Resende $\cdot$ Rui Campos
}

Received: 17 March 2009/Accepted: 17 March 2009/Published online: 14 August 2009

(C) The Author(s) 2009. This article is published with open access at Springerlink.com

\begin{abstract}
Sustainability must be seen as a global issue. In order to achieve that goal, it is necessary to apply its principles to all industrial activities, including those that are not traditionally engaged with such guidelines, which is the case of Bridge Construction. This paper evaluates the consumption of steel and energy and the emissions of carbon dioxide due to the use of movable scaffolding systems (MSS) in the Bridge Construction industry. The values obtained considering the use of conventional MSS are compared with the ones obtained using a new sustainable technology which is herein synthetically described-the organic prestressing system (OPS). In order to compare the sustainability of the two systems, a prediction of the material and energy consumptions, and $\mathrm{CO}_{2}$ emissions for traditional MSS and for MSS equipped with OPS is performed until 2025 .
\end{abstract}

\section{Introduction}

Plethora of bridge construction methods are currently used all over the world. The bridge construction method used in a specific bridge depends, among other issues, on the bridge characteristics (span length, type of deck, number of

P. Pacheco $(\varangle)$ · A. P. Adão da Fonseca · A. Resende

Bridge Engineering Research and Design (BERD),

Matosinhos, Portugal

e-mail: pedro.pacheco@berd.eu

P. Pacheco

FEUP, Porto, Portugal

R. Campos

Mota Engil Engenharia, Amarante, Portugal spans, etc.), the location of the bridge and the experience of both the contracting entity and the constructor.

One of the most used bridge construction methods is the cast in situ span by span construction with movable scaffolding systems (MSS). These equipments are large traveling steel structures that support the formwork that gives shape to the bridge. Depending on its characteristics, a traditional MSS can weigh up to more than 1,000 tons (mostly steel).

The use of MSS in bridge construction involves three main processes, which consume steel and energy and are responsible for the emission of carbon dioxide: manufacturing, transportation and operation.

Considering the significant dimensions of these equipments, their manufacture implies the use of considerable amounts of steel and energy, also producing important emissions of carbon dioxide.

The use of organic prestressing system (OPS) on MSS allows a significant reduction of steel needs-implying reductions of both energy consumptions and carbon dioxide emissions. On the other hand, this new technology implies additional energy consumption during its operation, due to the fact that OPS makes use of energy (instead of mass) to achieve stiffness.

After a brief presentation of the OPS concept and of its first full scale application, comparisons between the consumptions of steel and energy, and the emissions of carbon dioxide due to the use of MSS with or without OPS are presented.

\section{OPS technology—structural efficiency}

OPS is an innovative structural solution that resulted from a research and development process initiated in 1994, at the 
Faculty of Engineering of the University of Porto. Typical scientific main stages were followed: fundamental research (Pacheco and Adão Da Fonseca 1996; Pacheco 1999, 2002), numerical analysis (Pacheco and Adão Da Fonseca 1997, 2002; Pacheco et al. 2004; Guerra et al. 2004), experimental tests (André et al. 2006) and, more recently, a full scale application was implemented (Pacheco et al. 2007).

OPS is a concept inspired in the behavior of an organic structure found in nature: the muscle (Pacheco and Adão Da Fonseca 1996; Pacheco 1999, 2002). It is nothing else than an actively controlled prestressing system, in which the tension applied is automatically adjusted to the actuating loads, through a control system, in order to reduce structural deformations and minimize tensions.

Numerical studies of different OPS applications to civil engineering structures reveal that OPS can be very advantageous for structures with high "live-load/dead-load" ratios (Pacheco and Adão Da Fonseca 1997). Scaffolding systems are a good example of such structures, as the dead load (self weight of the MSS) is around 20\% of the weight of the live load (weight of fresh concrete).

The main structural advantages of OPS when applied to MSS are simple to identify. Regarding Fig. 1, the prestressing forces imposed by the cables are simultaneously applied with the pouring of the fresh concrete (live load), so that the beam with a main span $L$ assumes a structural behavior similar to a continuous beam with three times $\mathrm{L} / 3$ long span. Deflections and bending moments are substantially reduced. If a conventional prestressing was applied (previously) to the "empty structure" (Fig. 2), an undesirable behavior would occur-the prestressing effect would be, by itself, nearly as much adverse as the live-load (deck weight) effect.
The main elements of the OPS system are: (1) an actuator in the organic anchorage, (2) unbonded cables, (3) sensors and (4) an electronic controller in the girder control unit (Fig. 3).

The control strategy of the OPS system adopts the midspan vertical deflection as the primordial control variable. The mid-span deflection is measured by means of sensors (pressure transducers) strategically spread along the structure. To implement this technique, a reservoir is fitted in a fixed location (near a pier) and pressure sensors, spread along the structure and connected by a fluid circuit (Fig. 4), measure variations in the hydrostatic pressure (Pacheco et al. 2004; André et al. 2006).

The sensors transmit the information to an automaton, which processes it according to a control algorithm, and then "decides" between maintaining or changing the prestressing force (Pacheco and Adão Da Fonseca 1997; Pacheco et al. 2004). Typically, in a concrete pouring situation, the concrete pouring mode is turned on and, if the mid-span deflection exceeds a pre-defined limit, the automaton "decides" to increase the hydraulic jacks (actuator) stroke, moving the organic anchorage beam and simultaneously tensioning the prestressing cables.

In addition, OPS performs continuous monitoring of the main girder steel structure, evaluating the main structural parameters and emitting warnings or alarms in case of anomalous situations.

\section{Rio Sousa Bridge—-brief description of pilot} application

In 2005, the first full scale prototype of a MSS with OPS was implemented for the construction of the Rio Sousa
Fig. 1 Structural effects of OPS on a scaffolding structure

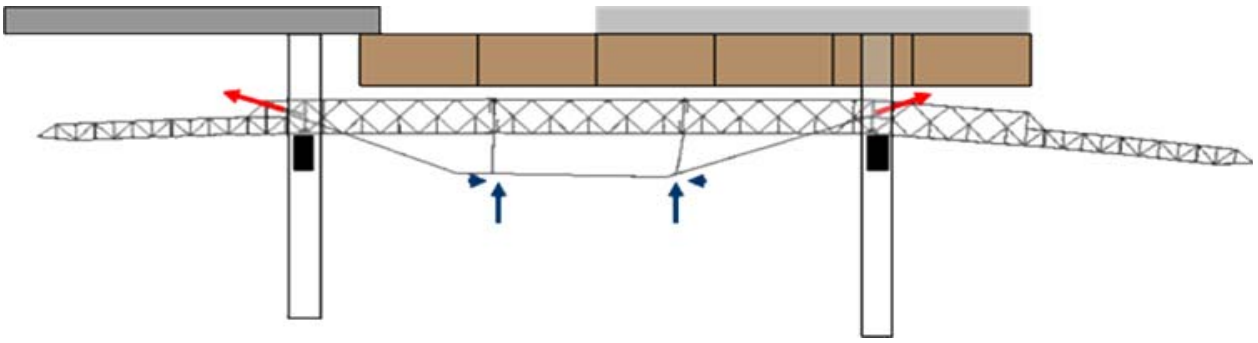

Fig. 2 Structural effects of conventional prestressing on a scaffolding structure

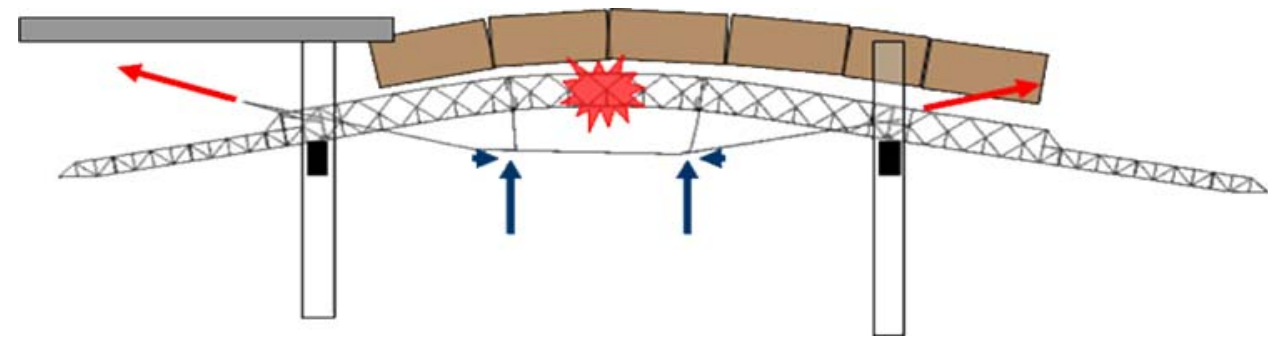


Fig. 3 3D scheme of a movable scaffolding system equipped with OPS (Pacheco et al. 2004)

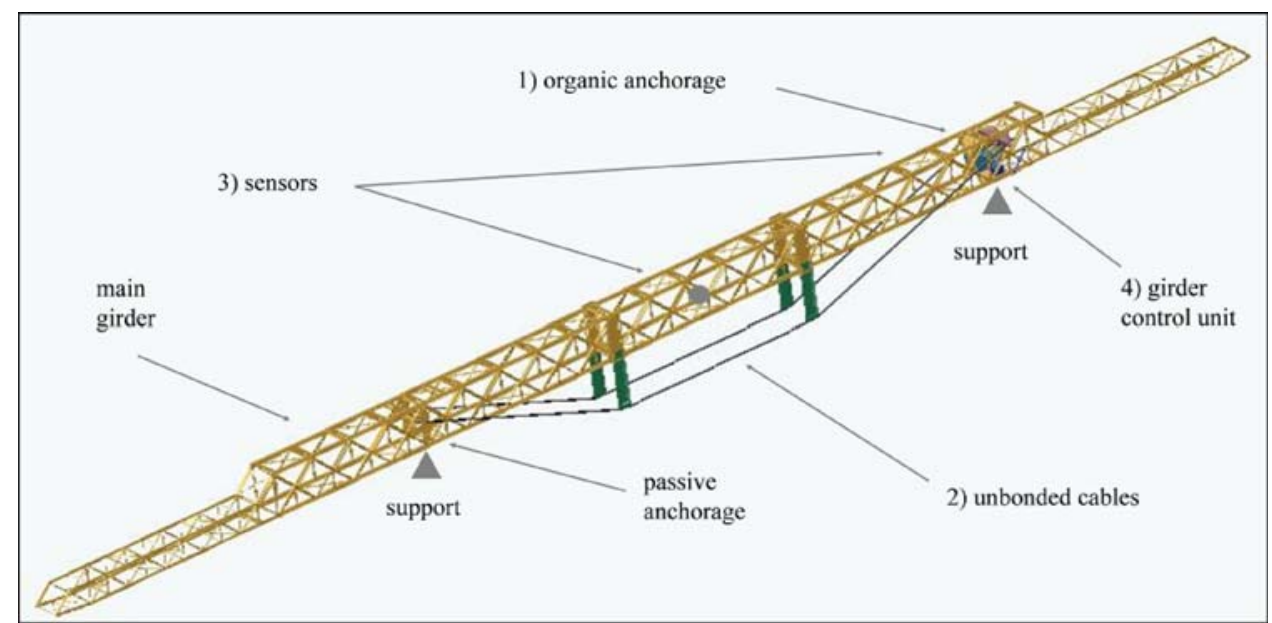

The main girders are modular trusses. Their transversal section $(1.25 \mathrm{~m} \times 2.00 \mathrm{~m})$ was designed for easy transportation and on site assemblage. The steel weight of the 4 main girders is approximately 108 metric tons (BERD and AFAssociados 2005). According to numerical studies, additional $30 \%$ of structural steel would be required in a conventional system for the same purposes (Guerra et al. 2004; Pacheco et al. 2007). Moreover, any conventional solution is unlikely to achieve such a high performance concerning deflection limitation.

\section{Why is OPS a clean technology?}

The use of the OPS technology in MSS allows a significant reduction of steel needs, implying reductions of both energy consumption and carbon dioxide emissions. However, OPS uses additional energy during its operation, due to the fact that it makes use of energy (instead of mass) to achieve stiffness.
Fig. 5 View of Rio Sousa Bridge

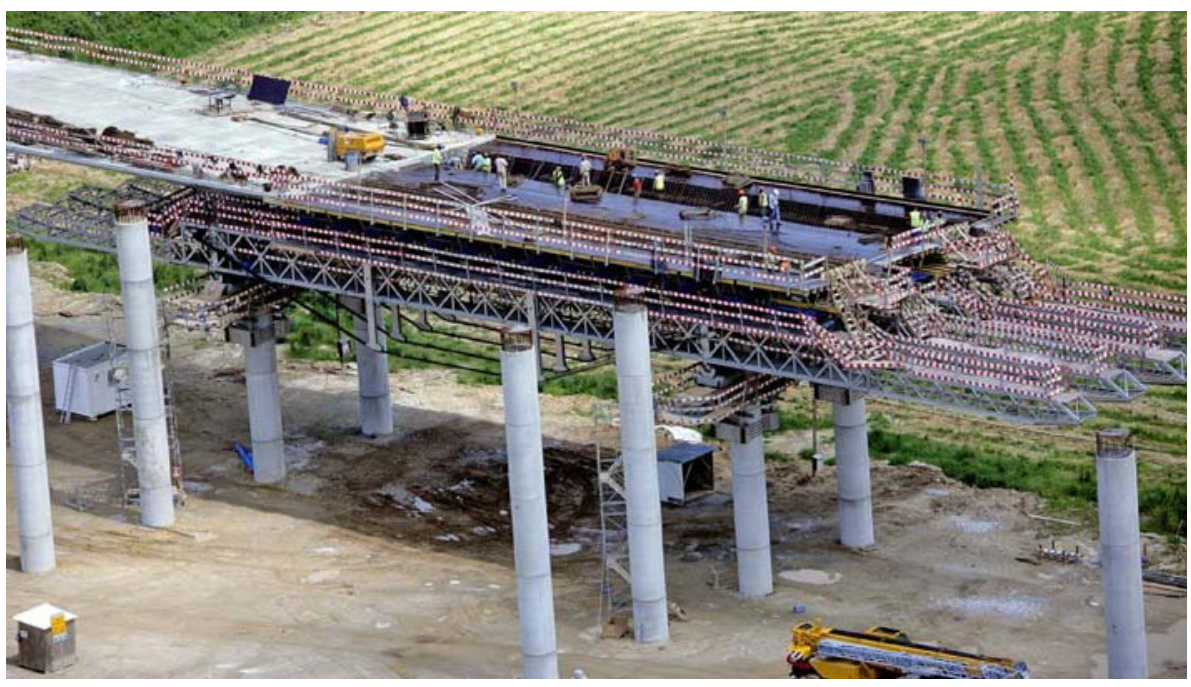


In order to evaluate the effect of the OPS technology in this industrial application, a systematic comparison is performed considering common life cycles of MSS. For simplicity, only the main processes are considered: manufacturing, transportation and operation on site.

\section{Steel consumption}

This analysis is made considering a representative MSS with 500 ton, which corresponds to the average weight of a traditional MSS (Moura 2005).

According to previous publications, the OPS system allows an average reduction of $30 \%$ in steel consumption (Guerra et al. 2004). Thus, the representative MSS with OPS technology weighs approximately 350 ton, resulting in nearly 150 ton saving per representative MSS unit.

\section{Energy consumption}

During their life cycle, MSS are responsible for a considerable amount of energy consumption, mainly during manufacturing and transportation processes.

In order to secure uniformity in the analysis, the Ton of Petroleum Equivalent (TPE) is adopted as the measure of energy consumption. The conversion between energy consumption and TPE is done according to the Portuguese Standard for the Regulation of Energy ConsumptionRGCE.

\section{Manufacturing}

Manufacturing includes the production of the raw material and assemblage of the MSS modules. According to RGCE, it is established that the energy consumption per metric ton of constructed steel is $0.51 \mathrm{TPE} / \mathrm{metric}$ ton (RGCE). Therefore, the energy consumption for the fabrication of one MSS without OPS is 255 TPE, which is to be compared with $181 \mathrm{TPE}$ for an equipment with OPS (179 TPE are due to the MSS structural steel structure manufacturing).

Manufacturing of the hydraulic equipment of the OPS corresponds to a reduced amount of energy consumption. Indeed, even considering that cold rolling steel manufacturing processes (used for hydraulic components manufacturing) represent an energy consumption per ton 2 to 3 times larger than the remaining steel components (Stubbles 2000), the weight of hydraulic equipment represents less than $0.5 \%$ of the MSS global weight (BERD and AFAssociados 2005). The same applies to the electronic equipment, which represents less than $0.05 \%$ of the MSS global weight (BERD and AFAssociados 2005).

\section{Transportation}

In a normal life cycle, main transportation operations occur "to site" and "from site". For simplicity partial transportations "to" and "from factory" are not herein considered.

For the evaluation of energy consumption during transportation, it is considered that, in average, a MSS has to travel an average distance of $300 \mathrm{~km}$ to be brought to site (Moura 2005), and it is assumed that only ground transportation with road trucks is used for the transportation of the equipment.

According to international transportation agents, a 26ton truck consumes 35 diesel liters per $100 \mathrm{~km}$, i.e., 0.01351 of diesel $/ \mathrm{km} / \mathrm{ton}$. The density of diesel is $835 \mathrm{~kg} /$ $\mathrm{m}^{3}$ and the conversion factor between the diesel consumption and TPE is 1.045 TPE/metric ton of diesel (RGCE), leading to $1.175 \times 10^{-5} \mathrm{TPE} / \mathrm{km} / \mathrm{metric}$ ton of steel.

Therefore, the energy consumption required for the transportation "to site" and "from site" of the representative MSS without OPS is 1.8 TPE, comparing to 1.2 TPE for the representative equipment with OPS.

\section{Operation}

The operation of a MSS involves multiple tasks that are very similar for equipments with or without OPS. Thus, only the energy consumption due to the OPS operation is considered in this analysis (differential quantification).

According to the first full scale application technical data, where a 170 ton equipment was used (BERD and AFAssociados 2005), OPS consumed approximately $3 \mathrm{~kW} \mathrm{~h}$ per hour of operation. Therefore, for the representative MSS with OPS technology, weighing 350 tons, the energy consumption by the OPS is predicted to be $6 \mathrm{~kW} \mathrm{~h}$ per hour of operation. Considering approximately $350 \mathrm{~h}$ of operations during the construction of a typical bridge deck, a consumption of about $2,100 \mathrm{~kW} \mathrm{~h}$ would be expected. Taking a conversion factor between $\mathrm{kW} \mathrm{h}$ and TPE of $290 \times 10^{-6}$ (RGCE), a consumption of 0.6 TPE is expected for the operation of a MSS with OPS.

\section{Energy consumption balance}

Considering the manufacturing, transportation and operation, the aggregated reduction of energy consumption achieved with OPS technology for the representative MSS is nearly 74 TPE (Table 1).

Carbon dioxide emissions

The carbon dioxide emissions analysis is performed only for manufacturing and for the transport operation. The 
Table 1 Steel and energy consumptions and $\mathrm{CO}_{2}$ emissions for the representative MSS units with and without OPS

\begin{tabular}{lll}
\hline & MSS without OPS & MSS with OPS \\
\hline Steel consumption (tons) & 500 & 300 \\
Energy consumption (TEP) & & \\
Manufacturing & 255.0 & 181.2 \\
Transportation & 1.8 & 1.2 \\
Operation & 0.0 & 0.6 \\
Total & 256.8 & 183.0 \\
Carbon dioxide emissions (tons) & \\
Manufacturing & 270.3 & 192.0 \\
Transportation & 4.9 & 3.4 \\
Total & 275.2 & 195.5 \\
\hline
\end{tabular}

operation stage is not relevant concerning carbon dioxide emissions.

\section{Manufacturing}

Although no specific information of MSS manufacturers is available, it is possible to estimate carbon dioxide $\left(\mathrm{CO}_{2}\right)$ emissions of steel manufacturing, considering average indicative values of this industrial sector. According to average values included in an OCDE/IEA report (OECD/ IEA 2007), a gross relation between $\mathrm{CO}_{2}$ emissions and energy consumption is $1.06 \mathrm{CO}_{2}$ ton/TPE. These values lead to an estimation of $\mathrm{CO}_{2}$ emissions of 270 ton for traditional MSS and of 192 ton for MSS equipments with OPS.

\section{Transportation}

The calculation of carbon dioxide $\left(\mathrm{CO}_{2}\right)$ emissions is performed adopting a density of $835 \times 10^{-6}$ metric ton/ diesel liter (RGCE). It is also considered that 1 metric ton of diesel produces 43.33 GJ (Global Reporting Initiative 2006) with an efficiency of $90 \%$, and finally, it is established that $1 \mathrm{GJ}$ of diesel emits $74.1 \mathrm{~kg}$ of $\mathrm{CO}_{2}$ (Portuguese National Inventory Report on Greenhouse Gases 1990).

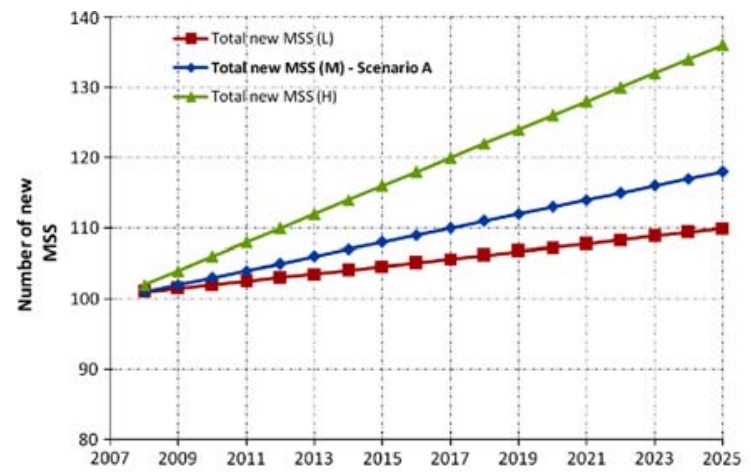

Therefore, the $\mathrm{CO}_{2}$ emission during transportation is 2.41 $\mathrm{CO}_{2} \mathrm{~kg}$ per liter of diesel. Multiplying this value by 0.0135 liters of diesel $/ \mathrm{km} / \mathrm{ton}$, a value of $0.0402 \mathrm{~kg}$ of $\mathrm{CO}_{2} / \mathrm{km} / \mathrm{ton}$ is obtained, that can be used to estimate the $\mathrm{CO}_{2}$ emissions due to the transportation of MSS "to site" and "from site". This results in 4.9 tons of $\mathrm{CO}_{2}$ emissions for a traditional system, compared with 3.4 tons of $\mathrm{CO}_{2}$ emissions for a system equipped with OPS technology.

\section{Rebuilt equipments}

Usually, MSS are used several times due to economic reasons, and normally each rebuilding process implies a partial addition of steel.

The average additional amount of steel used to rebuild a MSS is nearly $15 \%$ of the steel weight of the original MSS (Moura 2005). Thus, a representative rebuilt MSS is considered, admitting that it implies $15 \%$ additional consumption of steel and energy during the manufacturing process, and that it represents $115 \%$ additional consumptions and $\mathrm{CO}_{2}$ emissions, during the transportation process.

\section{The OPS effect-scenarios}

This study is based on scenarios taken from the available data of a marketing study for the MSS world market until 2025, developed by a MSS supplier (Moura 2005), that considers three different scenarios (low, medium and high) for the evolution of the number of new and rebuilt MSS in the world (Fig. 6) and for the implementation of OPS technology in MSS (Fig. 7).

For the evaluation of "the OPS effect" due to the introduction of the OPS technology in MSS, only the medium scenarios are considered. Therefore, scenario A considers only traditional MSS units without OPS (Fig. 6) and Scenario B is a predicted combination of both kinds of MSS, with and without OPS (Fig. 8).

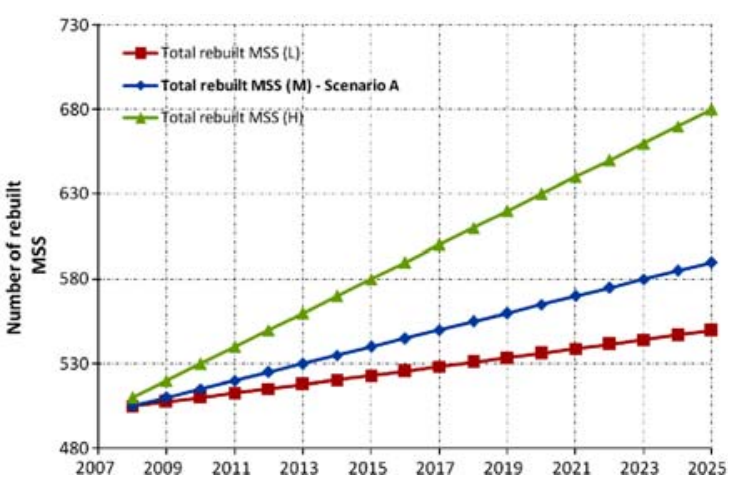

Fig. 6 Number of new and rebuilt MSS 

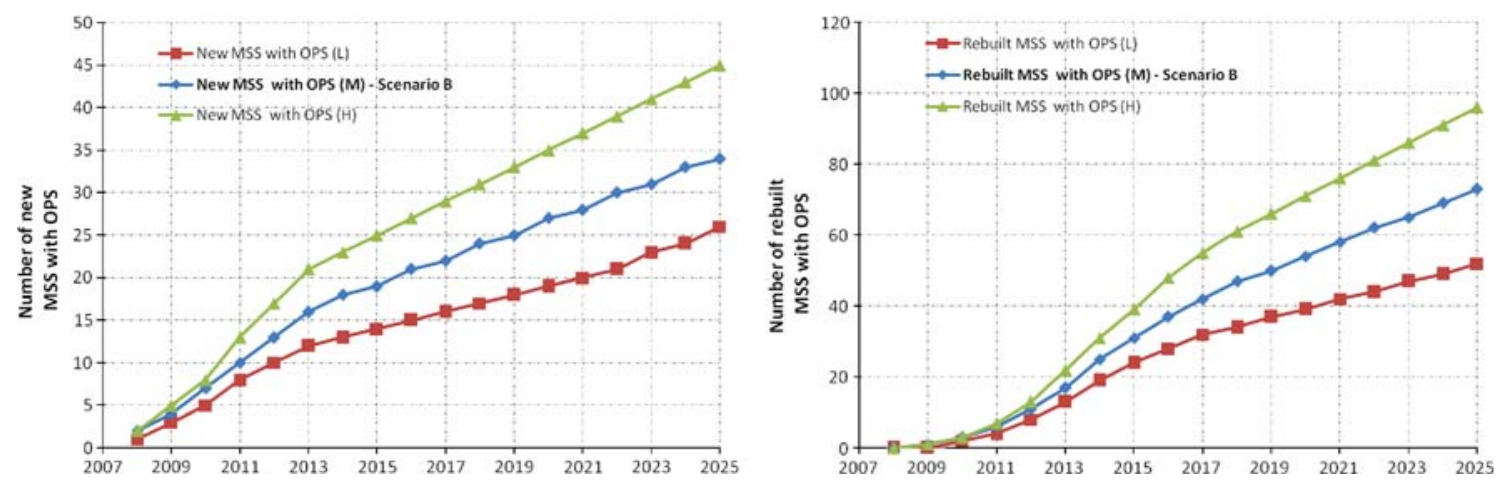

Fig. 7 Number of new and rebuilt MSS with OPS
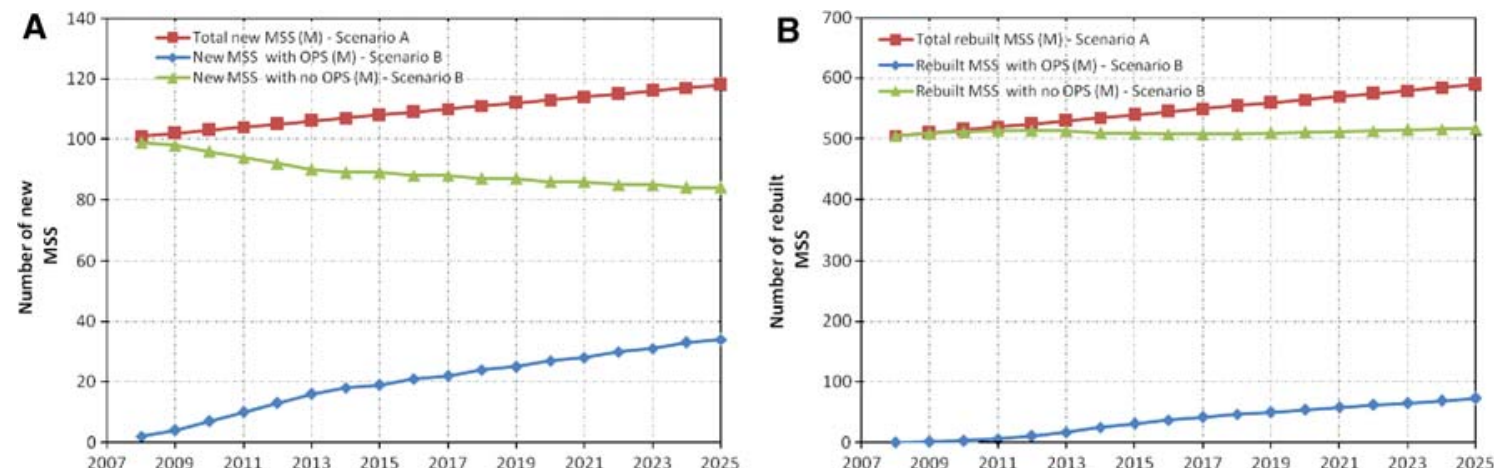

Fig. 8 Scenarios A and B for new and rebuilt MSS

According to the marketing document mentioned above, for Scenario A, 118 new MSS units and 590 rebuilt MSS units will be produced per year by 2025 . According to the same document, 34 new MSS units with OPS will be produced and 73 rebuilt ones will be used in 2025. Also according to Scenario B, 84 new MSS units and 517 rebuilt MSS units, both without OPS, will be used in 2025.

\section{The OPS effect-results}

Considering the above scenarios $\mathrm{A}$ and $\mathrm{B}$, the "OPS effect" is evaluated performing linear combinations of the global number of new MSS units and rebuilt ones, based on the above presented values of steel and energy consumptions, and $\mathrm{CO}_{2}$ emissions (Table 1), obtained for the representative MSS equipments with and without OPS.

If Scenario B occurs, in 2025, nearly 6.750 tons/year of steel consumption reduction would be achieved (Table 2). The reduction in steel consumption has an important economic impact on the fabrication of MSS, but it also implies reduction of energy consumption and emissions of $\mathrm{CO}_{2}$. Indeed, Scenario B implies a reduction of energy consumption of about 3,300 TPE/year (Table 3) and nearly 3,575 metric tons/year of $\mathrm{CO}_{2}$ emissions (Table 4).
Table 2 Steel consumption for scenarios A and B (metric ton)

\begin{tabular}{llll}
\hline & 2010 & 2015 & 2025 \\
\hline Scenario A (All MSS without OPS) & 90,125 & 94,500 & 103,250 \\
Scenario B & 89,008 & 90,953 & 96,508 \\
Saving & 1,117 & 3,547 & 6,742 \\
\hline
\end{tabular}

Table 3 Energy consumption for scenarios A and B (TPE)

\begin{tabular}{llll}
\hline & 2010 & 2015 & 2025 \\
\hline Scenario A (All MSS without OPS) & 47,189 & 49,479 & 54,061 \\
Scenario B & 46,640 & 47,739 & 50,755 \\
Saving & 549 & 1,740 & 3,306 \\
\hline
\end{tabular}

Table 4 Emissions of $\mathrm{CO}_{2}$ (metric tons)

\begin{tabular}{llll}
\hline & 2010 & 2015 & 2025 \\
\hline Scenario A (All MSS without OPS) & 52,109 & 54,639 & 59,698 \\
Scenario B & 51,514 & 52,740 & 56,083 \\
Saving & 595 & 1,898 & 3,614 \\
\hline
\end{tabular}

If cumulative curves are considered, much more expressive values are obtained. By 2025, total savings of about 70,000 ton of steel and 34,000 TPE of energy are achieved 

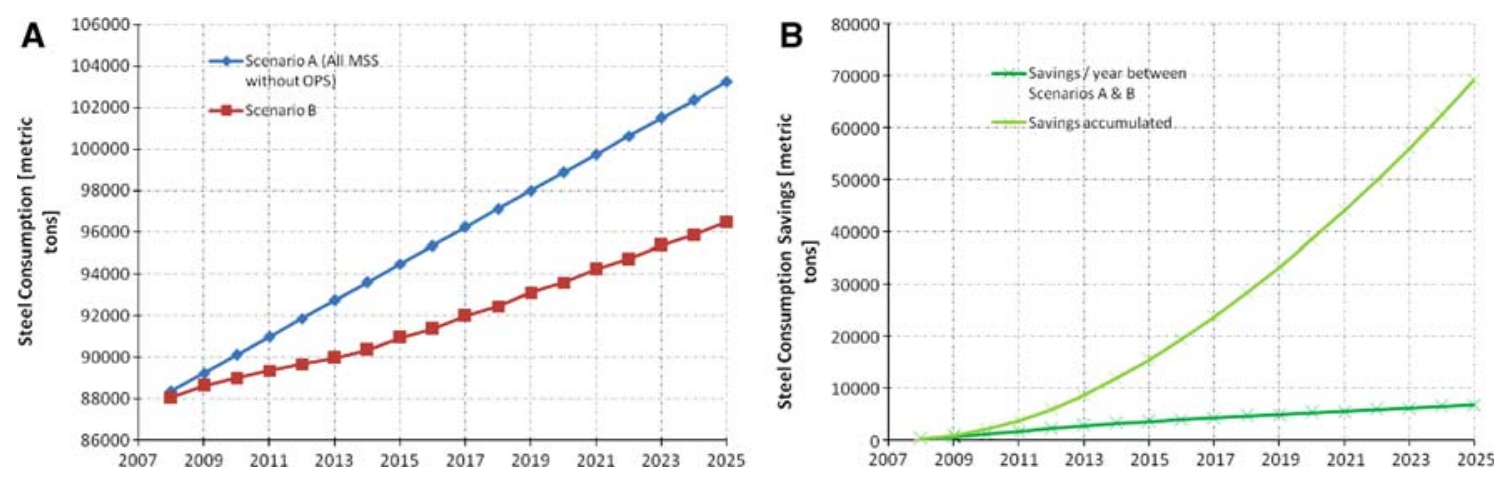

Fig. 9 Steel consumptions and savings for Scenarios A and B
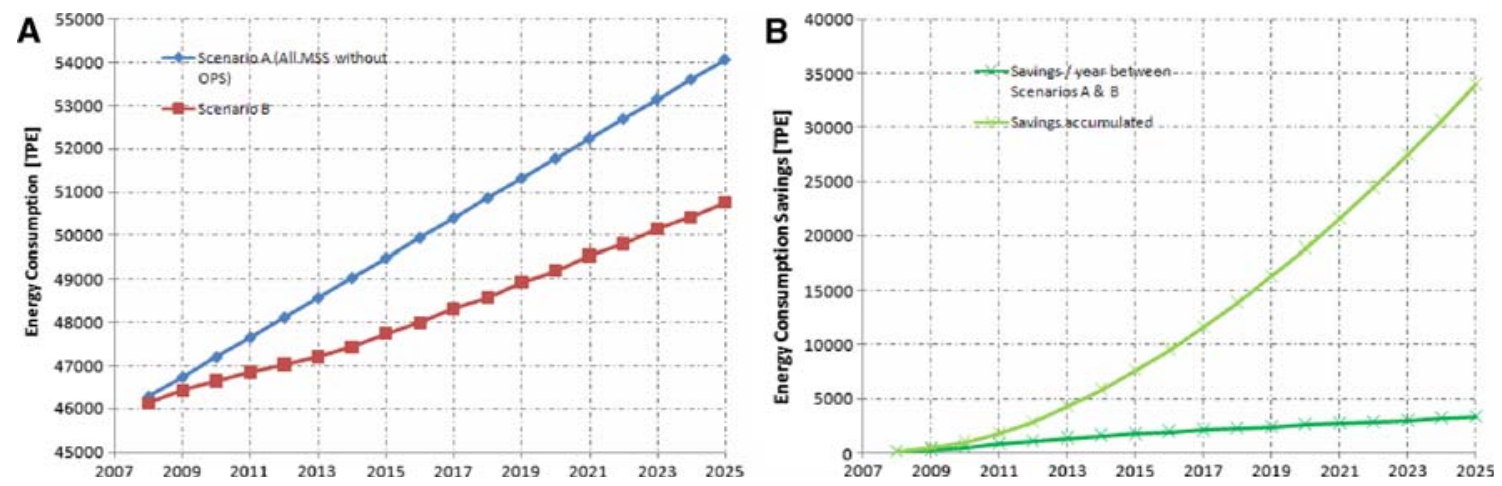

Fig. 10 Energy consumptions and savings for Scenarios A and B
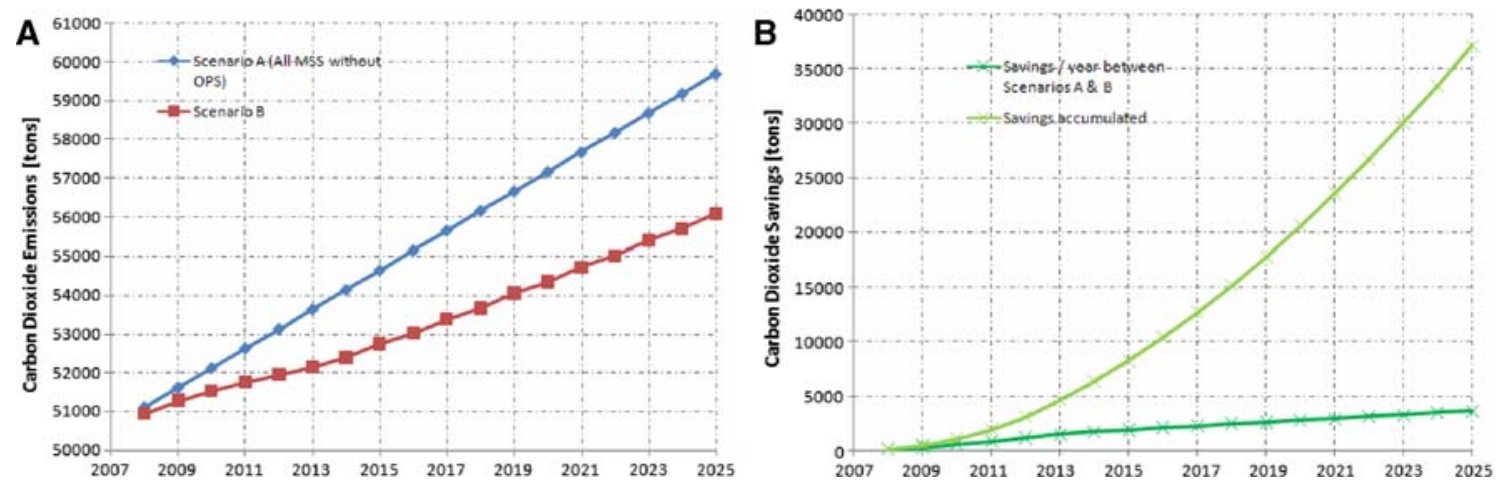

Fig. $11 \mathrm{CO}_{2}$ emissions and reduction for Scenarios A and B

(Figs. 9, 10), and a reduction of nearly 37,000 metric tons in the emission of $\mathrm{CO}_{2}$ is also achieved (Fig. 11).

\section{Conclusions}

Considering average scenarios, the introduction of OPS technology in the Bridge Construction industry shall

represent significant savings in terms of steel needs and energy consumptions. Also, it shall represent an important reduction of carbon dioxide emissions. According to numerical predictions, a reduction of $6.5 \%$ in steel consumption per year will be achieved in this specific industrial area in 2025 . A reduction of nearly $6 \%$ is predicted concerning both energy consumptions and Greenhouse gases emission. By 2025, that would mean a reduction of 6,750 
tons/year of steel needs; 3,300 TPE/year of energy consumption and 3,575 metric ton/year of $\mathrm{CO}_{2}$ emissions.

It is concluded that, besides the technical advantages, the OPS implementation contributes to the sustainability in bridge construction.

Moreover, the global savings could be even more important if contractors are encouraged to save energy and to decrease carbon dioxide emissions: the proportion of MSS with OPS would then become greater and mentioned benefits would increase.

Open Access This article is distributed under the terms of the Creative Commons Attribution Noncommercial License which permits any noncommercial use, distribution, and reproduction in any medium, provided the original author(s) and source are credited.

\section{References}

André A, Pacheco P, Adão Da Fonseca A (2006) Experimental study of a launching gantry reduced scale model strengthened with organic prestressing. Struct Eng Int 16(1):49-52

Berd, AFAssociados (2005) OPS movable scaffolding system—detail design (in Portuguese)

Global Reporting Initiative (2006)

Guerra A, André A, Pacheco P, Adão Da Fonseca A (2004) Organic prestressing-application on movable scaffolding systemsbasis of a pilot project (in Portuguese). Betão Estrutural 2004. In: Proceedings of GPPE symposium, Porto, 2004, vol 2, pp 1089-1096
Lisconcebe (2004) Rio Sousa Bridge-detail design (in Portuguese) Moura RG (2005) BERD business plan V01

OECD/IEA (2007) Tracking industrial energy efficiency and $\mathrm{Co}_{2}$ emissions

Pacheco P (2002) Organic prestressing - an example of an effector system, in structural concrete. J Fib 3(2):107-113

Pacheco P, Adão Da Fonseca A (1996) Effector systems in structures, conceptual design of structures. In: Proceedings of IASS symposium, Stuttgart, pp 339-346

Pacheco P, Adão Da Fonseca A (2002) Organic prestressing. J Struct Eng ASCE, 400-405

Pacheco P, Adão Da Fonseca A (1997) Organically prestressed multispan continuous box girders, new technologies in structural engineering. In: Proceedings of IABSE international conference, Lisboa, pp 527-534

Pacheco P (1999) Organic prestressing - an effector system example (in Portuguese). PhD Thesis, Department of Civil Engineering, Faculty of Engineering, University of Porto

Pacheco P, Adão Da Fonseca A, André A, Guerra A, Freitas F, Oliveira T, Pinto C, Mendes J (2004) Strengthening by organic prestressing of existing launching gantries, in the construction of high speed railway bridge decks. Workshop Bridges for HighSpeed Railways, Porto, pp 289-299

Pacheco P, Guerra A, Borges P, Coelho H (2007) A scaffolding system strengthened with organic prestressing - the first of a new generation of structures. Struct Eng Int 17(4):314-321 (published by IABSE)

Portuguese National Inventory Report on Greenhouse Gases 19902004, Instituto do Ambiente, Dezembro 2006

RGCE (Regulamento de Gestão do Consumo de Energia). Dec. Lei ${ }^{\circ}$ $58 / 82$ e Port. $359 / 82$

Stubbles J (2000) Energy use in the U.S. steel industry: a historical perspective and future opportunities. U.S. Department of Energy, Washington 